vol. 26 - $n^{\circ} 2 \mid 2010$

Numéro ouvert

\title{
Éric VIAL, L'Union populaire italienne, 1937-1940. Une organisation de masse du Parti communiste italien en exil
}

Ralph Schor

\section{(2) OpenEdition}

Journals

Édition électronique

URL : https://journals.openedition.org/remi/5170

DOI : $10.4000 /$ remi.5170

ISSN : $1777-5418$

Éditeur

Université de Poitiers

Édition imprimée

Date de publication : 1 septembre 2010

Pagination : 215-224

ISBN : 978-2-911627-55-2

ISSN : 0765-0752

Référence électronique

Ralph Schor, «Éric VIAL, L'Union populaire italienne, 1937-1940. Une organisation de masse du Parti communiste italien en exil », Revue européenne des migrations internationales [En ligne], vol. $26-n^{\circ} 2$ । 2010, mis en ligne le 08 décembre 2010, consulté le 15 avril 2022. URL : http:// journals.openedition.org/remi/5170; DOI : https://doi.org/10.4000/remi.5170 


\section{NOTES DE LECTURE}

\section{BARBA Serge}

De la frontière aux barbelés. Les chemins de la Retirada 1939. - Canet : Trabucaire, 2009. - $255 \mathrm{p}$. ISBN : 978-2-84974-086-6

$L$ 'afflux brutal des républicains espagnols fuyant la répression franquiste, afflux enregistré entre le 28 janvier et le 13 février 1939, a déjà inspiré de nombreuses études. Cette retraite où Retirada lança sur les chemins de l'exil quelque 500000 personnes, combattants et civils, valides et blessés, femmes, enfants, vieillards. Rien n'était préparé pour accueillir les réfugiés, bien que les autorités françaises eussent envisagé la possibilité d'un tel exode. Aussi l'accueil, si l'on peut employer ce terme, s'effectua-t-il dans les plus mauvaises conditions : improvisation, méfiance inspirée par les arrivants, brutalité parfois.

Serge Barba, président de l'Association des fils et filles de républicains espagnols et enfants de l'exil (FFREEE), a voulu reconstituer les itinéraires suivis par les réfugiés entre leur passage de la frontière et leur arrivée dans les camps dits alors de "concentration ». Le livre se présente comme une sorte de chronique évoquant la vie quotidienne au long de chaque chemin. Le récit est nourri par de nombreux documents, cartes, rapports officiels, photos d'époque, articles de journaux, témoignages, dessins et œuvres d'art diverses laissés par les acteurs de l'exode.

Le premier lieu de passage se situe à Cerbère où l'on arrivait par mer et surtout par terre. Les témoignages initiaux évoquent une situation qui se renouvellera en chaque point de la frontière: " une population affolée ", des "fuyards mal protégés par leurs haillons contre le froid», des lieux d'hébergement improvisés dans « des maisons inhabitées insalubres, des granges, des halles, des prisons désaffectées, d'anciens haras, d'anciens moulins... ». Puis sont passés en revue les autres points de passage, Banyuls, Port-Vendres où furent amenés des navires-hôpitaux, le Perthus, La Vajol-Las Illas, Saint-
Laurent-de-Cerdans, Lamanère, Prats-deMollo, Py-Mantet, la Cerdagne. Beaucoup de relations mentionnent la brutalité des forces de l'ordre, surtout les Sénégalais et les Spahis, et la bonne volonté des élus locaux ainsi que celle de certains ecclésiastiques comme l'abbé Bousquet, curé de Saint-Laurent-de-Cerdans.

Après avoir été contrôlés, fouillés, désarmés et filtrés, les républicains étaient vivement invités à regagner leur pays. Ceux qui restaient, étudiés dans la deuxième partie du livre, se trouvaient " entre frontière et camp ». Ils étaient rassemblés à Arles-sur-Tech, tête de pont ferroviaire des évacuations, Amélie-les-Bains, Céret, le Boulou, Perpignan où s'installèrent nombre de hautes personnalités de la république espagnole défunte.

La troisième partie est consacrée aux camps, Argelès, Saint-Cyprien, le Barcarès, Collioure, le Vernet d'Ariège, Agde, Rivesaltes... Beaucoup de lieux se révélaient impropres à leur fonction: Argelès où furent enfermées quelque 100000 personnes était situé sur une plage marécageuse. Des monographies mettent l'accent sur des lieux particuliers; la maternité d'Elne, le centre spécial de Rieucros en Lozère, le château de Collioure où les réfugiés jugés les plus dangereux étaient rassemblés dans des conditions de détention très dures.

Le livre apporte peu de révélations sur les camps, déjà très étudiés. Mais il reconstitue avec émotion l'expérience douloureuse vécue au quotidien par les Espagnols, les efforts généralement consentis avec humanité par les communes frontalières, l'incurie des autorités supérieures et parfois la brutalité des forces de l'ordre. Ce livre dans son entier constitue luimême une sorte de témoignage.

Ralph Schor Département d'Histoire Université de Nice-Sophia-Antipolis 


\section{SIMON BAROUTH Ida}

Juifs à Rennes, Étude ethnosociologique. - Paris, L'Harmattan, 2009. — 604 p. + cahier photographique de $23 \mathrm{p}$.

ISBN : 978-2-29610-755-7

$C$ Yet ouvrage propose une enquête ethnosociologique approfondie d'une petite collectivité : les familles juives de Rennes. Réalisée dans la perspective de la sociologie des relations interethniques, cette enquête minutieuse parvient à nous donner une connaissance approfondie de l'histoire et des formes d'appartenance de celles et ceux qui, aujourd'hui à Rennes, s'identifient à cette ethnicité juive, dans la diversité de ses appropriations individuelles et collectives, quotidiennes et festives.

S'appuyant sur un matériau impressionnant, nourri d'observation participative longue (plusieurs années) et d'entretiens nombreux et particulièrement approfondis, l'auteure nous fait entrer avec une certaine sensibilité dans les souvenirs, mais aussi dans les pratiques (culinaires, éducatives, religieuses, linguistiques, associatives, festives, etc.) qui fondent, jour après jour, le sentiment " d'appartenir 》 à un collectif et "d'éprouver " une identité. Tous ces récits constituent un matériau d'une grande richesse, permettant de saisir ce que Daniel Cefaï appelle les " contextes d'expérience et d'activité des acteurs ». Émaillant régulièrement ses descriptions et ses analyses de nombreux extraits d'entretiens, l'auteure prend le contrepied des travaux parfois rapides et peu fondés, où de rares extraits d'entretiens se contentent d'illustrer le raisonnement de l'auteur : ici, l'ethnologue prend au contraire très au sérieux les narrations que les femmes, les hommes, les jeunes adultes et les plus âgés lui font de leur propre trajectoire et de celle de leurs parents et grands-parents. Ils confient aussi leur vision des liens familiaux, les leçons qu'ils tirent du passé, de ces souvenirs, construisant avec l'ethnologue la mémoire de leur " groupe » ou, en tout cas, de ce milieu auquel ils se sentent appartenir plus ou moins fortement, certains depuis longtemps, d'autres depuis peu, leur sentiment d'appartenance à la judéité étant pour beaucoup le fruit d'un engagement individuel et collectif dans un processus de communalisation.

Comprendre aussi finement les contextes d'expériences, permet ensuite de saisir ce que peu d'enquêtes permettent de restituer, à savoir " des affectivités, des sensibilités, des imaginaires, des identités, des mémoires et des projets » (Céfaï, 2001). C'est bien finalement ce que parvient à faire Ida Simon-Barouh, adoptant un plan d'ouvrage (le passé puis le présent) qui privilégie la compréhension des individus, des familles et des associations sur un temps long, les décennies reconstituées permettant de comprendre d'une génération à l'autre, les logiques d'engagement (pour le religieux, pour la vie associative, pour la culture, etc.), mais celles aussi de distanciation vis-à-vis de pratiques ou de valeurs perçues comme éminemment juives.

Ce temps long, c'est aussi celui qui permet d'appréhender le sentiment d'appartenance de façon dynamique, en montrant que les évènements historiques, des conditions de vie nouvelles ou redéfinies, une transmission familiale défectueuse ou au contraire particulièrement accomplie d'une génération à l'autre, une trajectoire familiale où chacun, en vieillissant, prend conscience qu'il est un maillon d'une chaîne culturelle singulière, et bien d'autres dimensions sensibles sur plusieurs décennies, peuvent expliquer les attitudes d'aujourd'hui, les réticences à se dire juif en famille ou en public, ou au contraire l'assurance tranquille, mais néanmoins vigilante d'être un Juif aujourd'hui à Rennes.

L'ouvrage est composé de deux parties : une première traite, à partir des récits recueillis, de l'histoire familiale des quatre-vingt-dix personnes rencontrées, de leurs activités domestiques et religieuses, des "traditions " telles qu'elles étaient interprétées autrefois, des façons de se marier, de s'exprimer, d'élever les enfants, dans des circonstances qui ont souvent été celles de la migration, du déplacement ou de la fuite ; la seconde traite notamment de la vie collective aujourd'hui telle qu'elle s'est organisée autour d'associations cultuelle et culturelle, mais aussi de la transmission, de l'identité, des perceptions 
d'Israël et des modalités concrètes d'insertion dans la vie rennaise.

Outre le foisonnement d'informations qui donnent une image sinon complète tout au moins complexe de la vie de cette petite " communauté " (le terme y est discuté), l'ouvrage se termine par une réflexion approfondie sur l'identité ethnique et la situation minoritaire qui la nourrit. Construite à partir des points de vue des personnes rencontrées, la judéité qui se dessine au fil des pages apparaît comme une construction fragile, toute en finesse, parfois invisibilisée, parfois clairement revendiquée, entretenue par le regard (souvent réel, parfois supposé) du majoritaire, mais aussi par les souvenirs, la mémoire collective, l'histoire, les rites, les valeurs, les objets, les moments festifs, où l'entre-soi domine. Au-delà, toutefois, de l'expression individuelle de l'appartenance, le travail fouillé sur la vie associative montre combien les liens de type communautaire n'ont pas une vitalité intrinsèque, ne se maintiennent pas tout seuls. Ils impliquent au contraire un investissement important : celui offert naguère par ceux qui se sont mobilisés pour obtenir une salle de prière puis une synagogue, alors même que Rennes apparut longtemps comme un " désert » juif, et celui aujourd'hui de ceux qui se mobilisent régulièrement pour que la " communauté » existe et se pérennise à Rennes, pour que la ville constitue une terre accueillante aux nouveaux venus susceptibles de venir s'y installer.

L'ouvrage propose une anthropologie culturelle de ces familles sur plus de six cents pages : c'est le temps nécessaire pour s'imprégner des valeurs, saisir le sens des pratiques et peut-être même s'attacher, comme sut l'être certainement l'auteure, à ces individus et leurs familles, à leurs manières "d'être au monde" si singulières et en même temps tellement (et simplement)... humaines.

Hélène Bertheleu CNRS UMR 6173 CITERES Université de Tours
TARRIUS Alain et BERNET Olivier

Migrants internationaux et nouveaux réseaux criminels. - Canet : Trabucaire, 2010. - $160 \mathrm{p}$. ISBN : 978-2-84974-112-2

Chaque ouvrage de Tarrius enchaîne sur les conclusions du précédent, exposant donc une enquête sans fin, depuis 1985, sur la naissance, les évolutions et peut-être même la mort de cette forme migratoire qu'il dénomme la transmigration.

C'est encore le cas ici, mais à l'importante différence près que l'objet est moins l'identification des formes des nouvelles migrations internationales, les transmigrations, que leur absorption par de nouveaux réseaux criminels. Ils fédèrent les anciennes "pieuvres " italiennes, un pan entier des transmigrations caucasiennes, moyen-orientales et balkaniques, à des entreprises d'électronique asiatiques et à toutes sortes d'organisations policières, douanières et consulaires. Ainsi, ces réseaux sont à même de dresser une «toile » recouvrant la Méditerranée, l'Europe méridionale et ses entours moyenorientaux et caucasiens. Nous assistons, nous dit Tarrius, à une réorganisation des réseaux criminels sur le modèle de la " toile " Internet, c'est-à-dire " mondialisés, polyvalents, complémentaires, accessibles ", et en rupture avec les anciennes formes "mafieuses", tout en les survalorisant. Les chercheurs " déroulent" des trajectoires de migrants depuis Hérat, en Afghanistan, jusqu'aux «clubs » de prostitution du Levant espagnol, en passant par les Balkans et l'Italie du Sud.

Un nouvel ordre criminel se déploie, se globalise, sans contrôle, attirant bas et haut des échelles sociales, alors que les États peinent à définir leur place dans cette insaisissable mondialisation.

\section{La mondialisation des pauvres}

Deux types de réseaux et de circulations migratoires, qui se développaient parallèlement, avec plus d'évitements et de conflits que d'interférences et d'affinités, sont donc en train de 
fusionner à l'occasion des derniers avatars de la crise financière du monde globalisé.

- D’une part les vieux réseaux criminels, qui faisaient circuler les drogues, les armes et les femmes, suivant une logique territoriale de " pieuvre », c'est-à-dire des expansions omnidirectionnelles à partir de centralités micro localisées gérant de vastes territoires ; ils manifestaient cependant, dès lors qu'ils étaient menacés pour telle activité illicite, une forte capacité de fractionnement en micro unités opaques selon une logique de "famille ". L'observateur n'apercevait alors qu'une manifestation locale de cet ensemble criminel que l'on qualifie de cette notion très intermédiaire, floue et peu opératoire, de " mafieux».

- D'autre part les nouvelles transmigrations, ou un type récent de migrations internationales, où des centaines de milliers de petits entrepreneurs nomades se déploient en réseaux de revente avantageuse de produits d'usages licites non taxés, aux quatre coins de la planète, véritable force de travail transfrontalière commerciale des grandes firmes mondialisées, gênées dans leur expansion par les règles interétatiques du commerce officiel. Cette force de travail nomade réalise les stratégies commerciales des grandes firmes : le " poor to poor" "l'entre pauvres ", c'est-à-dire la vente par des pauvres de produits d'entrée de gamme à des populations réputées non solvables dans les conditions de taxations du marché international. Ces transmigrants, peu sensibles aux réglementations fiscales et commerciales des nations qu'ils traversent, effectuent en plusieurs mois des tournées, de chez eux à chez eux, et nulle part ne posent des problèmes d'intégration aux sociétés qu'ils côtoient, travaillant brièvement, afin d'assumer leurs déplacements, aux champs et dans le bâtiment après avoir écoulé leurs marchandises. Leurs clientèles privilégiées sont les populations pauvres des nations pauvres ou riches : poor to poor, par les pauvres et pour les pauvres est en effet un objectif commercial des grandes firmes dans leur déploiement mondial ${ }^{1}$. Là, les réseaux sont très lisibles, d'un bout à l'autre des distributions, et les marchandises commercialisées sont d'usage licite, mais évidemment échappent aux taxes usuelles et donc peuvent atteindre des populations à la solvabilité faible; pour l'essentiel ces produits ne sont pas contrefaits, leur technicité étant trop complexe et trop évolutive (ordinateurs, clefs USB, MP3, appareils photo numériques, caméscopes, etc.).

\section{«Invisibilité » des transmigrants ?}

Les responsables étatiques, aussi bien que ceux des grandes $O N G$, s'abstiennent de reconnaître la nouvelle et importante forme de migrations internationales que représentent les transmigrants, dans un contexte où leurs activités ne sont pas criminelles. Par ailleurs, les réseaux criminels se maintiennent dans un flou propice à toutes les affabulations relayées par la presse à sensations. En effet, il apparaît relativement plus urgent et davantage à portée de l'action publique de gérer les demandeurs d'asile et les sans papiers, que de reconnaître des populations en migration qui excèdent de toutes les frontières, ne demandent aucune intégration, et se présentent comme des sortes de "justiciers" du commerce international. Dans ces conditions, on comprend que le recouvrement actuel des réseaux de transmigrants par les réseaux criminels soit pour l'heure peu accessible aux analyses des transformations des modes d'organisation de la criminalité.

Pour les $O N G$, ces migrants-là ne relèvent pas de leur action'. Ils leur apparaissent comme non nécessiteux et très extérieurs aux problé-

\footnotetext{
1 C'est ainsi que tel fabricant asiatique de produits électroniques qui commercialisait jusqu'en 2008 des micro-ordinateurs au prix plancher de 420 euros (prix constructeur) et des caméscopes pour 320 euros propose aujourd'hui des gammes à partir respectivement de 160 et 112 euros... mais les réseaux usuels de commercialisation ne "suivent pas" les réductions de marges ainsi impliquées : il reste ces petits commerçants internationaux que sont les transmigrants, habitués depuis plusieurs années à commercialiser des clefs USB et autres MP3.
}

2 Nous ferons une exception pour la CIMADE de Montpellier qui, au contact des Marocains transmigrants a bien compris leur problématique et leurs besoins d'assistance. 
matiques de l'assistance juridique. Pourtant, les situations sanitaires et affectives des transmigrants sont très proches de celles des autres migrants internationaux de la misère ${ }^{3}$.

Pour le moins un pan de la réalité sociale et économique des migrations contemporaines est ignoré. C'est-à-dire que la capacité d'analyse de toutes les circulations migratoires s'en trouve affectée : en effet les chercheurs ont en tous lieux aperçu la porosité des diverses formes4. Mais cette reconnaissance implique une vision autrement dynamique des mouvements planétaires des populations en migration ${ }^{5}$. Les diverses formes sont articulées et la compréhension de chacune est tributaire de la connaissance des autres. Le problème concomitant c'est la facilité donnée ainsi à la nouvelle forme, qui associe de plus en plus intensément l'économie criminelle à la transmigration, de se développer. Le paradigme du "lieu» dans les sciences sociales, et celui de l'"identité " conçue comme préséance locale du sédentaire sur le voyageur, ont réduit la mobilité à un état évanescent, une notion profondément abstraite, transitoire, à un manque peu exploré ou au mieux à une trajectoire parcourue d'origine à destination de flux de populations : il n'existe pas de "sujet » de mobilité, l'être est construit, repéré, analysé, essentiellement comme sédentaire. Il y a longtemps que Tarrius réinvestit les espaces-temps de la mobilité, du déplacement, des interactions sociales qui les caractérisent, qui font continuité, donnent pouvoir au migrant sur l'immobilité historique et constitutive des dispositifs sédentaires des États nations, ou encore, pourrait-on dire, des assignations à sédentarité. Face aux prétendus désordres de

3 Résultats d'une recherche menée (2007-2009) par HasniaSonia Missaoui, Lamia Missaoui, Olivier Bernet, et Alain Tarrius, pour la MIRE-DREES : "Accès aux soins des transmigrants $»$.

4 C'est ainsi que dans les Balkans les informations sur les migrants de la misère en Grèce sont abondantes et celles sur les nombreux transmigrants qui passent en Bulgarie sont inexistantes.

5 Nous rappellerons la notion de "système migratoire " proposée par Gildas Simon, Emmanuel Ma Mung, etc. qui solidarise toutes les formes migratoires internationales pour la compréhension de chacune d'entre elles. la mondialisation, cette stabilité ancienne de l'ordre immuable du lieu se présente souvent aujourd'hui comme source d'opacité, d'incertitude.

\section{Une nouvelle et puissante forme migratoire criminelle}

L'évolution de la transmigration afghane, que Tarrius étudie depuis six années ${ }^{6}$, est caractéristique de ce mouvement. Cette forme migratoire internationale-là est née au début des années 2000 et fédère en plusieurs vagues des dizaines de milliers de migrants pendulaires, d'Afghanistan jusqu'aux Balkans et retour. Ces Afghans cumulent durant un, deux, ou parfois trois semestres des petits emplois agricoles avec le commerce de produits électroniques en provenance du Sud-est asiatique, et passés hors taxes par Dubaï ou Koweï-City, soi-disant destinations terminales. Ils se chargent de ces marchandises auprès de petits aéroports, à Trabzon, port turc de la Mer Noire, ou à Samson, ou encore à Poti, en Géorgie, et les commercialisent dans les Balkans, principalement en Bulgarie, donc dans la Communauté Européenne. Ces commerces de "fourmis » permettent d'écouler des marchandises à hauteur de six milliards de dollars environ. De grandes banques des Émirats, Bahreïn, Dubaï, Koweït-City, leur consentaient à partir de Trabzon, Samson, etc., où ils reçoivent les marchandises acheminées par les aéroports côtiers, des lignes de crédit d'un montant moyen de $40 \%$ de leurs achats. Dès le mois de décembre 2008, ces banques refusèrent de renouveler leurs crédits, l'opération étant illégale, puisque ces marchandises, hors taxes, ne pouvaient désormais, suivant les engagements des États, être réexportées des Émirats où elles arrivent en " destination terminale". La Crise conduisait notamment en effet à un durcissement des régulations bancaires dans le sens d'une adéquation des circulations monétaires aux règles politiques internationales de transfert des marchandises. Dès lors, des milieux turco-italiens troubles constituèrent des "petites sociétés » d'investissement, à partir de

6 TARRIUS Alain, La remontée des Sud. Afghans et Marocains en Europe méridionale, L'Aube, 2007. 
"placements » de 10000 à 20000 euros par cinquante à cent épargnants : inutile de dire qu'il s'agit de prête-noms pour de l'argent $d u$ blanchiment des dérivés du pavot à opium, morphine et héroïne... que les Afghans doivent désormais cultiver au cours de leurs tournées, s'ils veulent obtenir le moindre crédit de ces " petites sociétés » d'investissement. Ainsi, depuis une année la transmigration afghane est soumise, sous peine de disparaître, aux milieux criminels turco-italiens... pour aider des Turcs et des Géorgiens à implanter une industrie des psychotropes illicites, et les firmes internationales de l'électronique du Sud-est asiatique à commercialiser leurs produits. Cette articulation nouvelle d'activités qui grossissent l'influence des réseaux criminels a provoqué un regain d'autres trafics: celui des femmes est le plus visible. Les auteurs, après enquête le long $d u$ Levant espagnol, de la frontière française jusqu'à Malaga, possèdent plus de 140 adresses d'interlocutrices prostituées venant de différents pays du Caucase, des Balkans, et du pourtour méditerranéen, consommatrices de cocaïne oulet d'héroïne, et pour $40 \%$ d'entre elles ayant vécu une étape-initiation aux usages prostitutionnels de la cocaïne en Italie du Sud. Elles ont déclaré leur accord pour des entretiens sur leurs trajectoires. Sept cas, après une typification rigoureuse des trajectoires spatiales ont donné lieu à des enquêtes sur les lieux d'origine, les circonstances de la migration, les lieux étapes, et les transactions lors de l'arrivée dans les clubs prostitutionnels de la côte méditerranéenne espagnole.

Les dizaines de milliers de transmigrants afghans, qui jusqu'alors commettaient un délit fiscal, sont désormais les chevilles ouvrières de réseaux criminels en prêtant main-forte, pour des salaires dérisoires, à la tentative de culture du pavot somniferum en Turquie et en Géorgie et en blanchissant une partie de l'argent de ses transformations par l'achat des produits électroniques à livrer en Europe. Les mouvements pendulaires de leurs transmigrations sont désormais liés aux phases culturales $d u$ pavot requérant l'intervention de " maind'œuvre spécialisée ", si possible étrangère, et nombreuse. Ils provoquent la corruption de nombreux représentants des États, précisément dans les lieux de passage des produits et des personnes : douaniers, policiers, agents consulaires en premier lieu.

\section{Un espace de rationalisation des circulations et valeurs des psychotropes}

Ouest Bulgarie, Macédoine, Sud Serbie, Albanie et Italie du Sud forment une vaste zone d'interpénétration entre la cocaïne, très présente dans l'Ouest européen, avec le "grand arrière " américain, et l'héroüne très présente dans le Moyen-Orient, récemment implantée dans le Caucase et l'extrême Est turc, avec "le grand arrière » asiatique. Dans ce vaste espace, les circulations des psychotropes sont gérées par les milieux criminels italiens, fortement implantés en Albanie, et Bulgares, très proches des Serbes ; des quantités de l'un et l'autre psychotrope, complémentaires dans les consommations, sont échangées et les valeurs monétaires du gramme sont soigneusement étalonnées afin de maintenir les revenus maximaux de chacun. C'est ainsi que le gramme d'hérö̈ne afghane de bonne qualité, négocié à dix euros à Trébizonde (Trabzon-Turquie) est à cinquante euros à Milan, Marseille ou Barcelone après passage de cette zone. La cocaïne d'une qualité négociée à trente euros le gramme à Barcelone se revend à soixante-dix euros en Bulgarie et au-delà. Ces "régulations " maintiennent la paix lors des échanges entre réseaux, rendant possibles des circulations de consommateurs (i.e. prostituées consommatrices des deux produits) et de revendeurs. Connaître les rationalités qui président à la fixation des prix et les contraintes qui permettent de faire respecter ce "marché des valeurs " est de première importance. L'évolution de l'assistance moyen-orientale à la lutte des Albanophones contre les Serbes, comme la fusion des milieux criminels albanais et sud italiens, donnent quelques clefs de compréhension présentées comme autant de pistes qu'une prochaine recherche permettra d'approfondir et de nuancer. 


\section{Vers de nouvelles formes}

Ce que l'on peut apercevoir des morphologies des nouveaux réseaux criminels, sous réserve de recherches plus précises, c'est d'une part leur fragmentation en itinéraires multiples non conflictuels et d'autre part leur ethnicisation et leur "démocratisation", dans le sens d'une plus grande accessibilité des réseaux criminels par les populations locales.

- Fragmentation en itinéraires multiples non conflictuels

De l'Europe de l'Est jusqu'à la France et l'Espagne on peut distinguer au moins six réseaux : celui qui, partant des ex-républiques soviétiques d'Asie, passe par la Pologne et l'Allemagne ; celui qui, partant du Caucase, passe par la Géorgie et l'Ukraine, puis l'Autriche et la Suisse ; celui qui, partant d'Asie du Sud-est, passe par le Golfe, le Liban, la Grèce, l'Italie du Nord et la France, est " animé " par des Chinois. Et trois autres sur lesquels ont porté ces recherches : celui qui, partant de Géorgie et d'Afghanistan, passe par la Turquie, l'Italie du Sud et l'Espagne; celui qui, partant d'Afghanistan, passe par la Turquie, la Grèce, le Sud de l'Italie, la France et l'Espagne ; celui qui, partant du Liban, traverse les pays sud-méditerranéens jusqu'en Espagne ; nous n'envisageons pas là les réseaux remontants (i.e. Nigéria, Maroc, France, Grande-Bretagne, ou encore Somalie, Libye, Italie du Sud) ni transatlantiques (Colombie, Brésil, Europe et Afrique, etc.). Il s'agit là des réseaux véhiculant cocaïne, crack, héroïne, morphine et brown sugar et des femmes pour des activités prostitutionnelles. La représentation cartographique de ces réseaux suggère une toile, à la façon de celle représentant Internet dans son déploiement mondial. Évidemment, cette multiplicité vit sans arrêt des chevauchements et irrigue souvent les mêmes régions. L'offre est ainsi diversifiée, tant en qualité qu'en prix, et elle couvre au mieux les territoires. On est passé d'un commerce de petits épiciers rivaux à une offre de type grands magasins multimarques, même si localement les revendeurs vivent çà et là des conflits commerciaux. Quant aux classiques formations criminelles, italiennes par exemple, elles occupent désormais des points de passage clefs, des carrefours des circulations, confortant, sans perdre leur vocation locale, leur nouveau rôle dans les déploiements criminels mondiaux : par exemple les jeunes femmes des Balkans, du Caucase ou des espaces proche-orientaux circulant vers les accueillants " clubs 》 du Levant espagnol effectuent généralement une étape en Italie du Sud afin de s'initier au commerce de la cocaïne lié à la prostitution.

Il n'y a plus de grands conflits entre réseaux. Cette évolution a été concomitante chez les transmigrants, comme dans les réseaux criminels. Il serait toutefois opportun d'identifier et de décrire des lieux de rencontre des réseaux et de comprendre les règles qui président aux diverses négociations et transactions. Notamment une vaste zone entre Sud Serbie et Sud Italie est celle de l'interpénétration de la cocaïne et de l'hérö̈ne : fixation des équivalences poids/valeurs monétaires, détermination des usages mixtes et spécifiques. Là encore, nous avons cru comprendre que les auteurs ont réservé cette investigation pour une prochaine recherche.

\section{- Ethnicisation}

Depuis une quinzaine d'années ${ }^{7}$ les chercheurs ont constaté que les transmigrants fonctionnent en réseaux ethniques et ont la particularité de développer un code d'honneur, un métalangage commercial, débarrassé des références religieuses ou idéologiques des uns et des autres, qui non seulement évite les conflits, mais de plus met en synergie la diversité pour l'obtention et la distribution d'un produit. Cette caractéristique qualifie aujourd'hui les nouveaux réseaux criminels. Chaque réseau possède la qualification de ses membres, Géorgiens obstinés, Chinois habiles commercialement, etc., ainsi que ses territoires d'implantation, mais sans que cela dépasse les représentations populaires usuelles, et de façon même à valoriser les uns et les autres. Tous ces réseaux " ethniques 》 sont solidaires face à leur nécessité de contourner la diversité des politiques des États concernant les étrangers et face à la nécessité de partager un

7 TARRIUS Alain et MISSAOUI Lamia, Arabes de France dans l'économie souterraine mondiale, L'Aube, 1995. 
immense marché. À Trabzon, l'offre d'héroïne se décline en termes de provenances diverses ("la russe ", "l'afghan ", "la turque ", "l'indienne ", "la chinoise », etc.), et les cours évoluent selon ces provenances, sans que les critères de différenciation soient clairs : chaque revendeur du quartier du port peut indifféremment fournir la diversité des produits, mais encore des commerçants russes ou afghans, etc., peuvent fournir exclusivement "leur" marchandise. Le tout sans conflits.

\section{- Démocratisation}

Ce ne sont plus des individus spécialisés dans le travail de rabatteurs qui recrutent clients et complices, mais des personnes attirées par les consommations, ou l'opportunité de gains faciles, qui ouvrent un petit marché de voisinage de psychotropes en s'adressant au réseau le plus proche ou le plus visible. De même pour la prostitution, c'est l'occasion qui provoque l'intervention d'un loueur d'appartements ou d'un tenancier de "boîte 》 dans l'espace méditerranéen et balkanique, auprès d'un gérant de club espagnol. Chacun de ces nouveaux partenaires peut fournir une entrée dans les différents réseaux criminels où participent de plus en plus d'individus aux profils sociaux « irréprochables, banals ». La règle de l'« omerta » se trouve particulièrement affaiblie par cette labilité des dépendances ou appartenances. Les chercheurs en ont fait l'expérience dans leurs enquêtes. L'accès à un terrain qui était complexe s'en trouve ainsi considérablement simplifié.

De plus en plus les "carrières " de prostitution, en Espagne, sont enclavées, durant quatre à sept ans, dans une histoire de vie familiale et sociale qui banalise ce " moment" en faisant continuité entre l'avant et l'après-prostitution ${ }^{8}$. Cela caractérise les femmes originaires des Balkans et du Caucase. Pour nombre d'entre elles, il semblerait cependant qu'après des années de pratique de "la passe avec la dose

8 Nos enquêtes sur les clubs espagnols de prostitution en 2008 et 2009 dénombrent $70 \%$ de retours dans les familles balkaniques après quatre à sept ans de prostitution. Voir TARRIUS Alain et BERNET Olivier, Migrants internationaux et nouveaux réseaux criminels, Rapport INHES 2009, à paraître en 2010. de coke ", l'usage de l'héroüne, très accessible dans leurs régions d'origine, se chronicise. Le retour, si fréquent, des prostituées de l'Est vers leur milieu social d'origine est marqué pour plus de $50 \%$ d'entre elles, selon les évaluations des chercheurs (enquêtes dans des clubs espagnols), par l'importation du VIH, du VHC et de l'addiction à l'hérö̈ne.

Lamia Missaoui Laboratoire Printemps

Université de Versailles Saint-Quentin-enYvelines

VIAL Éric

L'Union populaire italienne, 1937 1940. Une organisation de masse du Parti communiste italien en exil. - Rome : École française de Rome, 2007. — 461 p.

ISBN : 978-2-72830-756-2

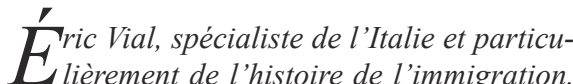
a tiré de son mémoire d'habilitation à diriger des recherches un remarquable ouvrage sur l'Union populaire italienne (UPI).

L'UPI naquit en 1937, à l'époque où les partis communistes sortaient de leur isolement et cherchaient à constituer de vastes rassemblements antifascistes, comme ce fut le cas en France en 1934-1936. Le PC italien offrit à l'UPI une aide matérielle, un financement provenant sans doute du Komintern, des locaux, une presse, un personnel d'encadrement, une base militante. De la sorte, la nouvelle organisation apparaissait d'emblée comme le satellite d'un PCI hégémonique qui la contrôlait et voulait l'utiliser pour recruter. Au congrès de fondation, à Lyon, les délégués communistes étaient trois fois plus nombreux que les autres antifascistes.

L'UPI offrait divers services à ses membres. Elle les aidait dans leurs relations avec l'administration française. Elle distribuait des secours alimentaires. Elle proposait des colonies de vacances. Elle mit sur pied des associations filiales destinées à rassembler divers groupes tels que les anciens combattants, les femmes, les jeunes. Elle organisait des activités culturelles, 
sportives, ludiques comme, à Waziers dans le Nord, le concours du "plus gros mangeur de spaghettis sans les mains ". L'UPI fit aussi campagne pour appuyer une revendication de la gauche immigrée, la définition d'un statut juridique des étrangers.

Le discours de l'Union populaire se voulait unitaire et largement "interclassiste », ce qui la conduisait à effacer les références prolétariennes. En effet l'UPI tendait explicitement la main aux petits et moyens propriétaires, patrons, commerçants, aux paysans, aux professions intellectuelles et libérales, aux cadres, aux catholiques et même aux "frères en chemise noire ». Certains dirigeants semblent avoir cru à une possibilité de libéralisation $d u$ régime fasciste. Pour mieux fonder son aspiration unitaire, l'UPI exaltait la fierté de l'identité italienne. À cette fin elle montrait la beauté et la richesse de la langue et de la culture péninsulaires. Les artistes du passé comme les créateurs contemporains étaient régulièrement invoqués, ainsi Dante, Léonard de Vinci, Giotto, Michel Ange, Goldoni, Pirandello, d'Annunzio, Chirico, etc. Garibaldi et Mazzini étaient placés en tête des références historiques. Le rassemblement était aussi étayé par un éloge de la nation et de ses intérêts primordiaux, comme l'indépendance. Le caractère positif conféré à l'italianité permettait d'accepter une certaine intégration des immigrés, mais non leur assimilation. Enfin, l'UPI rejetait l'antisémitisme fasciste, contraire à la tradition italienne.

Les dirigeants populaires affichaient leurs sentiments francophiles, d'autant plus vibrants que la Front populaire, les grèves de 1936, les lois sociales, l'essor contemporain du syndicalisme rendaient le pays d'accueil particulièrement sympathique. L'amitié et la collaboration avec les hôtes français étaient facilitées par les choix démocratiques, antifascistes, pacifistes faits par le gouvernement de Paris. Le multipartisme et la démocratie, même dans son incarnation américaine, étaient vantés. Mais l'UPI, sans crainte de la contradiction, célébrait également la forme soviétique de la démocratie. Le rejet de l'impérialisme fasciste, de l'Axe, de la conquête de l'Éthiopie, de Franco apparaissait très ferme. Dans le domaine culturel, l'UPI, tout en promouvant le réalisme socialiste, se voulait moderne, ouverte aux recherches et au progrès, soucieuse d'élever le niveau des masses.

L'organisation de l'UPI, dotée seulement de trois permanents au niveau national, resta légère. La direction était cependant ferme et centralisée. Les sections locales avaient surtout pour mission de recruter, d'animer, de diffuser le message du sommet et d'obtenir un consensus minimal. Le nombre des adhérents évolua sans doute entre 30000 et 40000 au grand maximum. Des sections purent être implantées dans des régions de faible immigration italienne, mais la vitalité fut surtout notable dans le Sud, la région Rhône-Alpes et une bande départements allant du Nord au Doubs. Le tirage du journal de l'UPI, la Voce degli Italiani, avoisinait les 15000 exemplaires.

Les ambiguïtés du discours tenu par l'Union populaire entravèrent son développement. En effet, la francophilie était contredite par la politique du gouvernement Daladier qui surveillait fermement les étrangers et leur imposait de nombreuses contraintes. L'exaltation de l'identité italienne s'accordait mal avec l'intégration de plus en plus forte des immigrés et les naturalisations, fréquentes même chez les dirigeants du mouvement. Les positions unitaires conduisant à tendre la main à des catégories très éloignées de la gauche étaient mal reçues par une base attachée au militantisme rouge traditionnel. À partir de 1938, les effectifs reculèrent tandis que les prises de position classiques de la gauche prenaient plus de corps. La cohésion interne de l'UPI était mal assurée : certains communistes trouvaient l'organisation trop modérée et les non-communistes condamnaient le poids $d u$ PCI. Si le rejet des trotskystes était général, les méfiances et les querelles étaient fréquentes.

Les tensions internationales forcèrent l'UPI à préciser la nature de sa francophilie. L'organisation, certes pacifiste, fut conduite par sa solidarité pour le pays d'accueil à promettre de s'engager les armes à la main pour défendre celui-ci. Lorsque Mussolini réactiva ses reven- 
dications irrédentistes, l'UPI se plaça aux côtés de la France et organisa des engagements de volontaires. À l'approche de la guerre, les communistes resserrèrent leur emprise sur l'UPI. La signature du pacte germano-soviétique traumatisa l'Union et entraîna sa disparition rapide. Certains dirigeants essayèrent d'abord de minimiser l'importance du pacte. Mais les communistes, condamnant les démocraties et les socialistes, s'alignèrent sur Moscou. Le principal animateur de l'UPI, Cocchi, hostile au pacte, fut exclu du PCI. Les socialistes, outrés par le ralliement des révolutionnaires à l'accord germano-soviétique, s'éloignèrent de l'UPI ou essayèrent localement d'en évincer les staliniens. En tout cas, l'activité s'effondra et l'Union disparut dans le tourbillon de la guerre.

Éric Vial montre in fine que l'UPI joua un faible rôle dans les secteurs où l'intégration des immigrés était très avancée et connut la réussite dans le cas inverse. Mais, par son discours de synthèse mariant sentiment national italien et francophilie, elle facilita l'assimilation : elle montrait qu'un étranger peut prendre de nouvelles racines sans renier les anciennes. Cette thèse est développée avec brio dans un livre savant, mais non pesant. L'érudition, étayée par un appareil critique très riche, apparaît impressionnante. La démonstration, la nuance dans le jugement et l'interprétation entraînent l'adhésion du lecteur. Existe-t-il des livres définitifs? Si oui, celui-ci en constitue un parfait exemple.

Ralph Schor Département d'Histoire Université de Nice-Sophia-Antipolis 